\title{
MODELS OF SEXUAL AND NATURAL SELECTION IN POLYGAMOUS SPECIES
}

\author{
PETER O'DONALD \\ Department of Genetics, University of Cambridge, Milton Road, Cambridge CB4 1XH
}

Received 7.ix.72

\begin{abstract}
SUMMARY
The distinction between sexual selection and assortative mating is discussed. It is argued that assortative mating might evolve as a result of sexual selection. Simple models of sexual selection show that stable polymorphisms can be established by alleles with a wide range of additive and dominant effects. In more general models, the sexual selection is determined by female mating preferences depending partly on the frequencies of the preferred genotypes. However, if the mating preferences are directly proportional to the genotypic frequencies, there is no resulting selective effect on the population. When natural selection opposes the sexual selection, the ratio of the proportion of females exercising the mating preference to the natural selective coefficient determines whether a stable polymorphism can be established or not. Semler's experiments show that male sticklebacks who develop the typical red throat colour in the breeding season have a considerable sexual advantage over those who do not develop the colour. But they are at a selective disadvantage from predators. The frequency of the red males fits the model of a recessive gene maintained by a balance of sexual and natural selection.
\end{abstract}

\section{INTRODUGTION}

IN some papers published recently (O’Donald, 1972a, b, c) I have described the results of mathematical models of sexual selection in monogamous species. The models were first discussed by Darwin (1871). He suggested that sexually favoured males who mate first will gain an advantage since the first females to breed are likely to be in better condition and thus rear more offspring. Selection may be acting in this way on the polymorphism in plumage of the Arctic Skua, a sea-bird of the North Atlantic (O'Donald, 1972c). Darwin's proposed model should apply in general to animals with distinct breeding seasons.

Darwin knew very well, however, that sexual selection would be much more powerful when acting on males in a polygamous species: the favoured males would gain a great selective advantage if they were able to mate with many females. Extreme examples of sexual dimorphism, in which the males have very exaggerated characteristics for sexual display, are often to be seen in polygamous species of birds. Models of sexual selection in polygamous species show that the selection will be faster if a recessive gene is selected than if a dominant gene is selected (O'Donald, 1963, 1967). If the selection is the result of a female mating preference for certain male phenotypes, genetic factors determining the mating preference will also be selected (Fisher, 1930; O’Donald, 1967). Stable polymorphisms can be set up if natural selection opposes the sexual selection.

In this paper I shall describe the results of a more general model of sexual selection than my previous models. The model is more general because it 
allows for given proportions of the females to have mating preferences for each of the three male genotypes determined by two alleles at a locus. At the same time natural selection with either additive or dominant effects on the fitnesses of the genotypes is also incorporated in the model. The model is similar in many ways to some models of assortative mating analysed by Karlin (1969). Assortative mating can indeed be confused with sexual selection for it also involves mating preferences for particular genotypes. The distinction between these two phenomena should be clearly recognised.

\section{Sexual selection and assortative mating}

Sexual selection occurs in polygamous species when some males have a better chance of mating than others. In monogamous species it occurs when some males have a better chance of mating with the fitter females who are able to produce more offspring. The males who are favoured may be superior in fighting to maintain their harem or territory or by the females having a mating preference for them. Like natural selection, sexual selection may be directional, stabilising or disruptive. In terms of possible mating preferences, sexual selection will be directional if the females prefer one particular male phenotype which differs from the mean of the others; it will be stabilising if the mean phenotype is preferred; and it will be disruptive if the females have mating preferences for more than one of the male phenotypes.

Sexual selection is distinguished from assortative mating by the fact that, in sexual selection, the mating preferences are not determined by the genotypes preferred. In positive assortative mating a female with a particular genotype has a tendency to mate with males of the same genotype: her mating preference is determined by her own genotype at the locus of the genotypes she prefers in the males. In sexual selection the locus which is the object of the mating preference does not determine which genotypes are preferred. Thus if the females' mating preferences produce disruptive sexual selection for $A A$ and $a a$ males, the particular preference of a female for $A A$ or $a a$ males is not determined by whether the female herself is $A A, A a$ or $a a$. And indeed, in many cases of sexual selection, the genotypes of the $A$ locus will be sexlimited and not expressed in the females, for they will determine some character of sexual display limited to the males. In positive assortative mating, however, the female mating preference for $A A$ males will be expressed only in females who are themselves $A A$, for the essential feature of assortative mating is precisely that an individual's genotype determines his or her mating preference for or against others of that genotype. Assortative mating thus requires, in addition to the mating preference, a mechanism by which the preferred genotypes determine the preference. Kalmus and Maynard Smith (1966) showed that the behavioural mechanism of imprinting may be a cause of assortative mating in some polymorphisms, for example in the polymorphism of Chen caerulescens (Gooch and Beardmore, 1959). Pleiotropy could always be invoked as an hypothetical mechanism in any particular case but it can hardly be a general one.

More interesting is the possibility that assortative mating might evolve as a result of sexual selection. Models can be set up to show how a mating preference may evolve. As suggested by Fisher (1930), individuals who have an inherited tendency to mate with other advantageous individuals will themselves gain an advantage since their mating preference genes will be 
passed on to their offspring who will also on average possess the advantageous character. Since the mating preference itself adds to the advantage of the preferred character, a " runaway process", as Fisher called it, develops. Mathematical models (O'Donald, 1967) have confirmed that mating preference genes will be selected as a result of the inevitable correlation which develops between them and the genes that determine the advantageous character. The earlier the mating preference is established for the advantageous character, the greater is the correlation between the loci involved and the faster the selection. If the mating preference arises when the advantageous genes are rare, the selection eventually becomes very fast and the gene for the mating preference ends at a high frequency.

This correlation between the loci could lead to some degree of assortative mating. Suppose under disruptive selection both $A A$ and $a a$ are advantageous, and separate loci determine mating preferences for either $A A$ or $a a$ individuals. The gene for the mating preference for $A A$ will tend to segregate with $A$. Thus some assortative mating may be shown of $A A$ individuals for $A A$ individuals. However, the degree of assortative mating superimposed on the sexual selection would be slight unless the correlations were very high. And the correlations could only become high if the mating preference gene came to be incorporated into a supergene with the gene for the character preferred. There would be some selective advantage in the formation of such a supergene since it would minimise the occurrence of matings likely to produce disadvantageous genotypes. The optimum arrangement would be to have each allele that determined some advantageous character in close linkage with the alleles that determined the particular mating preference in its favour. The mating preference would then appear to be determined by the pleiotropic effects of the gene for the preferred character: assortative mating would have evolved as a result of the original disruptive selection. This is the process of evolutionary divergence leading to sympatric speciation. But according to this theory, mating preferences first evolve to produce disruptive sexual selection. The assortative mating then evolves as a result of an association between the mating preference and the particular gene preferred, thus leading to the evolution of a supergene and full assortative mating of the genotypes.

\section{The Model of SEXUAL SELEction}

In this model I shall ignore the problem of how the mating preference may be determined genetically and just assume that a certain proportion of the females will mate with males of a particular genotype.

\section{(i) Constant mating preferences}

I shall assume that $\alpha, \beta$ and $\gamma$ are the constant proportions of the females mating with $A A, A a$ and $a a$ males respectively, while the remaining females mate at random. Thus if $u, v$ and $w$ are the frequencies of the genotypes $A A$, $A a$ and $a a$, then the different mating types are formed with the following frequencies.

$$
\begin{array}{lc}
\text { Mating } & \text { Frequency } \\
A A \times A A & \alpha u+(1-\alpha-\beta-\gamma) u^{2} \\
A A \times A a & \beta u+\alpha v+2(1-\alpha-\beta-\gamma) u v
\end{array}
$$




$$
\begin{array}{ll}
A A \times a a & \gamma u+\alpha w+2(1-\alpha-\beta-\gamma) u w \\
A a \times A a & \beta v+(1-\alpha-\beta-\gamma) v^{2} \\
A a \times a a & \gamma v+\beta w+2(1-\alpha-\beta-\gamma) v w \\
a a \times a a & \gamma w+(1-\alpha-\beta-\gamma) w^{2}
\end{array}
$$

In the next generation the frequencies of $A A, A a$ and $a a$ will have become

$$
\begin{aligned}
u^{\prime} & =\alpha p+\frac{1}{2} \beta p+(1-\alpha-\beta-\gamma) p^{2} \\
v^{\prime} & =\alpha q+\gamma p+\frac{1}{2} \beta+2(1-\alpha-\beta-\gamma) p q \\
w^{\prime} & =\gamma q+\frac{1}{2} \beta q+(1-\alpha-\beta-\gamma) q^{2}
\end{aligned}
$$

where $p=u+\frac{1}{2} v$ and $q=w+\frac{1}{2} v$, being the gene frequencies of $A$ and $a$. Thus in the next generation $A$ has the gene frequency

or putting

$$
\begin{aligned}
p^{\prime} & =\frac{1}{2} \alpha+\frac{1}{4} \beta+p\left(1-\frac{1}{2} \alpha-\frac{1}{2} \beta-\frac{1}{2} \gamma\right) \\
\phi & =\alpha+\frac{1}{2} \beta \quad \theta=\alpha+\beta+\gamma \\
p^{\prime} & =\frac{1}{2} \phi+p\left(1-\frac{1}{2} \theta\right) .
\end{aligned}
$$

This is a generalisation of the recurrence equation for the sexual selection of a recessive gene (O'Donald, 1962) in which $\beta=\gamma=0$ and $\phi=\theta=\alpha$. Since in general

$$
\Delta p=\frac{1}{2} \phi-\frac{1}{2} \theta p
$$

at equilibrium

$$
p_{e}=\phi / \theta,
$$

which is obviously stable since $d(\Delta p) / d p$ is always negative.

Since in terms of $\phi$ and $\theta$, the genotypic frequencies are

$$
\begin{aligned}
u^{\prime} & =\phi p+(1-\theta) p^{2} \\
v^{\prime} & =\phi q+(\theta-\phi) p+2(1-\theta) p q \\
w^{\prime} & =(\theta-\phi) q+(1-\theta) q^{2}
\end{aligned}
$$

at equilibrium they have become

$$
\begin{aligned}
u_{e} & =p_{e}^{2} \\
v_{e} & =2 p_{e} q_{e} \\
w_{e} & =q_{e}^{2} .
\end{aligned}
$$

The genotypic frequencies are therefore in the Hardy-Weinberg ratios. This illustrates the distinction I have drawn between sexual selection and assortative mating: assortative mating produces an excess of homozygotes if positive and an excess of heterozygotes if negative when compared to the numbers expected from the Hardy-Weinberg ratios.

If the gene frequency of $A$ is $p_{0}$ at the start of selection, then after $n$ generations of sexual selection its frequency is

$$
p_{n}=(\phi / \theta)\left\{1-\left(1-\frac{1}{2} \theta\right)^{n}\left(1-\theta p_{0} / \phi\right)\right\},
$$

which demonstrates general convergence to the equilibrium $p_{e}$.

Cases of particular interest are when the genotypes are additive in terms of mating preference and $\beta=\frac{1}{2} \alpha, \gamma=0$. Then $\theta=\frac{3}{2} \alpha, \phi=\frac{5}{4} \alpha$ and $p_{e}=\frac{5}{6}$. When $A$ is recessive $\theta=\phi=\alpha$ and $p_{e}=1$ so that $A$ becomes fixed. When $\beta=0$ and there is disruptive sexual selection $p_{e}=\alpha /(\alpha+\gamma)$. 
When $A$ is dominant for the preferred male character, a different model is required: the females cannot then distinguish between $A A$ and $A a$ males in exercising their choice. In this model $A$ is again fixed by sexual selection (O'Donald, 1963).

\section{(ii) Frequency-dependent mating preferences}

The model of constant mating preferences is unrealistic when a genotype which certain females prefer is very rare. It is unlikely, if there were just one male of a particular genotype, that none the less a constant proportion of all females would still all mate with that one male. The mating preference might be frequency-dependent or might be partly frequency-dependent and partly independent of frequency. Suppose we put

so that

$$
\begin{aligned}
& \alpha=a+k p^{2} \\
& \beta=b+2 k p q \\
& \gamma=c+k q^{2}
\end{aligned}
$$

Then since

we have for this model

$$
\begin{aligned}
\theta & =a+b+c+k \\
\phi & =a+\frac{1}{2} b+k p . \\
\Delta p & =\frac{1}{2} \phi-\frac{1}{2} \theta p
\end{aligned}
$$

so that

$$
\Delta p=\frac{1}{2} a+\frac{1}{4} b-\frac{1}{2}(a+b+c) p
$$

$$
p_{e}=\left(a+\frac{1}{2} b\right) /(a+b+c) \text {. }
$$

The frequency-dependent component of the mating preference has no effect: if $a=b=c=0$, then obviously $\Delta p=0$, and nothing happens. For a more general model of frequency-dependent sexual selection we may put

$$
\begin{aligned}
& \alpha=a+k p^{2} \\
& \beta=b+2 l p q \\
& \gamma=c+m q^{2} .
\end{aligned}
$$

If the alleles are additive in their effects, then $m=c=0, b=\frac{1}{2} a$ and $l=\frac{1}{2} k$. Thus $\theta=\frac{3}{2} a+k p, \phi=\frac{5}{4} a+\frac{1}{2} k p(1+p)$ which gives $\Delta p=\frac{5}{8} a-\frac{3}{4} a p+\frac{1}{4} k p q$ and hence a quadratic equation for the equilibrium values of $p$. If $a=0$ and there is no constant mating preference value, then $\Delta p=\frac{1}{4} k p q$ and the simple frequency-dependent mating preference produces fixation of $A$ if $k$ is positive and fixation of $a$ if $k$ is negative.

Other frequency-dependent models can easily be suggested. For example the mating preference might be dependent on gene frequency and not on genotype frequency. For such a model we could put

$$
\begin{aligned}
& \alpha=a+k p \\
& \beta=b+\frac{1}{2} k p+\frac{1}{2} l q \\
& \gamma=c+l q .
\end{aligned}
$$

The solutions are easy to obtain for any particular case.

\section{The effegts of Natural selegtion in the Model}

In general, when very elaborate sexual displays have evolved as a result of sexual selection, they will ultimately reduce the chances of survival. The 
extreme development of plumage for sexual display in the male peacock or pheasant must surely make an escape from predators more difficult. It must surely make the birds easier to spot. Natural selection may be expected to. oppose the sexual selection of these characters. If the relative fitnesses of the genotypes $A A, A a$ and $a a$ are $1-s, 1$ and $1-t$, then the equations for the genotypic frequencies after the natural selection of the offspring produced by the matings which took place according to the model of sexual selection are as follows.

$$
\begin{aligned}
T u^{\prime} & =(1-s)\left\{\phi p+(1-\theta) p^{2}\right\} \\
T v^{\prime} & =\phi q+(\theta-\phi) p+2(1-\theta) p q \\
T w^{\prime} & =(1-t)\left\{(\theta-\phi) q+(1-\theta) q^{2}\right\},
\end{aligned}
$$

where $T=1-s \phi p-t q(\theta-\phi)-(1-\theta)\left(s p^{2}+t q^{2}\right)$. These equations give the difference equation

$$
T \Delta p=\frac{1}{2} \phi-\frac{1}{2} \theta p-p q\{s \phi-t(\theta-\phi)+(s p-t q)(1-\theta)\} .
$$

In this model, the natural selection is assumed to affect genotypes of both males and females together. If it only affects the males, then the genotypic frequencies of males and females will be different at mating time and this must be allowed for in the calculation of the frequencies of the different mating types.

(i) Sexual selection in favour of a recessive gene and natural selection against it

In this model $t=0$ since both $A a$ and $a a$ genotypes have the same fitness while $A A$ has a fitness of $1-s$. Since the mating preference favours only $A A$, therefore $\theta=\phi=\alpha$ since $\beta=\gamma=0$. Thus we have

$$
\Delta p=\frac{q\left(\frac{1}{2} \alpha-s \alpha p-s(1-\alpha) p^{2}\right)}{1-s \alpha p-s(1-\alpha) p^{2}} .
$$

There is a polymorphic equilibrium given by the expression for the frequency of $A$

$$
p_{e}=\frac{\alpha}{2(1-\alpha)}\left\{\sqrt{1+\frac{2(1-\alpha)}{s \alpha}}-1\right\} .
$$

This gives a simple expression for the phenotypic frequencies at equilibrium after mating has taken place, for

$$
u_{e}=\alpha / 2 s
$$

which is the phenotypic frequency of " A ". The frequency of the dominant phenotype "a" is therefore $1-\alpha / 2 s$. Polymorphic equilibria occur when $0<\alpha / 2 s<1$. They are stable equilibria: if $x$ is a small deviation from equilibrium, the deviation $x^{\prime}$ in the next generation can be shown to be

$$
x^{\prime}=x\left\{1-\frac{s \alpha}{(1-\alpha)(2-\alpha)} \sqrt{1+\frac{2(1-\alpha)}{s \alpha}}\left[(2-\alpha)-\alpha \sqrt{1+\frac{2(1-\alpha)}{s \alpha}}\right]\right\}
$$

and $x^{\prime}<x$ if

$$
2-\alpha-\alpha \sqrt{1+\frac{2(1-\alpha)}{s \alpha}}>0,
$$

which gives $\alpha / 2 s<1$ as the condition for stability. 
If the natural selection affects only the males, the recurrence equations of the model must be derived in a different way, for after natural selection the frequencies of the males have changed but not those of the females. Therefore it is necessary to allow for the effects of natural selection before sexual selection takes place. If in one generation the zygotic frequencies of the genotypes $A A, A a$ and $a a$ are $u, v$ and $w$ as before, then at mating time the frequencies in the females will still be $u, v$ and $w$, but in the males they will be

$$
\frac{u(1-s)}{1-s u}, \frac{v}{1-s u} \text { and } \frac{w}{1-s u}
$$

since selection has acted against the $A A$ males. The different mating types then have the following frequencies.

$$
\begin{array}{ll}
\text { Mating type } & \text { Frequency } \\
A A \times A A & \alpha u+\frac{(1-\alpha)(1-s) u^{2}}{1-s u} \\
A A \times A a & \alpha v+\frac{(1-\alpha)(2-s) u v}{1-s u} \\
A A \times a a & \alpha w+\frac{(1-\alpha)(2-s) u w}{1-s u} \\
A a \times A a & \frac{(1-\alpha) v^{2}}{1-s u} \\
A a \times a a & \frac{2(1-\alpha) v w}{1-s u} \\
a a \times a a & \frac{(1-\alpha) w^{2}}{1-s u} .
\end{array}
$$

The difference equation in terms of the gene frequency $p=u+\frac{1}{2} v$ is

$$
\Delta p=\frac{\frac{1}{2} q(\alpha-s u)}{1-s u}
$$

so that at equilibrium immediately after mating

$$
u_{e}=\alpha / s \text {. }
$$

The effect of the sexual selection is therefore twice as great as when natural selection affects both males and females. This is as it should be; since, in simple models, natural selection of one sex only has half the effect of selection of both sexes.

For $u$ at equilibrium we have the equation

$$
u_{e}=\frac{\alpha p_{e}-s u_{e} p_{e}+(1-\alpha) p_{e}^{2}}{1-s u_{e}}
$$

and putting $u_{e}=\alpha / s$ this immediately gives $p_{e}=\sqrt{\alpha / s}$. Then for a small deviation $x$ from $p_{e}$, we obtain the recurrence equation

$$
x^{\prime}=x\left\{1-\frac{\alpha}{(1-\alpha)}\left[\frac{1}{\sqrt{\alpha / s}}-1\right]\right\}
$$


showing that the equilibrium will be stable if

or

$$
\frac{1}{\sqrt{\alpha / s}}>1
$$

$$
\alpha / s<1 \text {. }
$$

(ii) Sexual selection in favour of a dominant gene and natural selection against it

If the preferred male character is determined by a dominant gene, the females will not be able to distinguish between the genotypes $A A$ and $A a$ when exercising their preference. Thus a different model must be used to determine whether a polymorphism can be maintained when natural selection opposes the sexual selection. If the natural selection affects only the males, a simple expression, identical to that for a recessive, can be derived for the equilibrium frequency of the preferred male phenotype. The derivation is as follows. After natural selection, we let the frequencies of the genotypes $A A, A a$ and $a a$ be $u, v$ and $w$ in the females and $u /(1+t w), v /(1+t w)$ and $w(1+t) /(1+t w)$ in the males. The mating types then have the frequencies

$$
\begin{array}{ll}
\text { Mating type } & \multicolumn{1}{c}{\text { Frequency }} \\
A A \times A A & \frac{\alpha u^{2}}{u+v}+\frac{(1-\alpha) u^{2}}{1+t w} \\
A A \times A a & \frac{2 \alpha u v}{u+v}+\frac{2(1-\alpha) u v}{1+t w} \\
A a \times a a & \frac{\alpha u w}{u+v}+\frac{(1-\alpha)(2+t) u w}{1+t w} \\
A a \times A a & \frac{\alpha v^{2}}{u+v}+\frac{(1-\alpha) v^{2}}{1+t w} \\
A a \times a a & \frac{\alpha v w}{u+v}+\frac{(1-\alpha)(2+t) v w}{1+t w} \\
a a \times a a & \frac{(1-\alpha)(1+t) w^{2}}{1+t w}
\end{array}
$$

This gives the difference equation

$$
\Delta p=\frac{\frac{1}{2} w p(\alpha+\alpha t-t+t w)}{(1-w)(1+t w)} .
$$

And hence at equilibrium

$$
w_{e}=1-\alpha\left(\frac{1+t}{t}\right) .
$$

In this model the preferred males have a fitness of 1 compared to the others whose fitness is $1+t$. In terms of a selective coefficient $s$, measuring the 
relative disadvantage of the $A$ phenotypes, these fitnesses would be $1-s$ and 1 respectively. Thus we can put

$$
t=\frac{\mathrm{s}}{1-s}
$$

so that

$$
\frac{1+t}{t}=\frac{1}{s}
$$

and hence

$$
w_{e}=1-\alpha / s .
$$

This is the frequency of the $a$ phenotypes at equilibrium. The frequency of the preferred $A$ phenotypes is therefore

$$
u_{e}+v_{e}=\alpha / s \text {. }
$$

These phenotypic frequencies are identical to those when sexual selection favours a recessive which is disadvantageous in natural selection; for then, as we have shown,

$$
\begin{aligned}
u_{e} & =\alpha / s \\
v_{e}+w_{e} & =1-\alpha / s .
\end{aligned}
$$

At equilibrium, the genotypic frequencies are $u_{e}=p_{e}^{2}, v_{e}=2 p_{e} q_{e}$ and $w_{e}=q_{e}^{2}$, but when sexual selection favours the recessive $p_{e}=\sqrt{\frac{\alpha}{s}}$, whereas for the dominant $p_{e}=1-\sqrt{1-\frac{\alpha}{s}}$. These equilibria are stable.

(iii) Sexual and natural selection of a gene with an additive effect on fitness

When the gene $A$ has an additive effect on fitness, we can put $t=-s$. Thus the fitnesses as a result of natural selection are $1-s, 1$ and $1+s$. If the natural selection affects both sexes, then the difference equation becomes

$$
\Delta p=\frac{\frac{1}{2} \phi-\frac{1}{2} \theta p-s p q}{1-s \phi+s \theta p-s(p-q)} .
$$

Since $A$ is additive in its effects, we have, as before, $\beta=\frac{1}{2} \alpha, \gamma=0$ and $\phi=\frac{\delta}{4} \alpha, \theta=\frac{8}{2} \alpha$ or $\phi=\frac{5}{6} \theta$. Thus at equilibrium

and

$$
s p^{2}-s p-\frac{1}{2} \theta p+\frac{5}{12} \theta=0
$$

$$
p_{e}=\frac{1}{2}\left\{1+\frac{1}{2}\left(\frac{\theta}{s}\right)-\sqrt{1-\frac{2}{3}\left(\frac{\theta}{s}\right)+\frac{1}{4}\left(\frac{\theta}{s}\right)^{2}}\right\} .
$$

As $s$ becomes relatively smaller and the ratio $\theta / s$ becomes large, this solution tends to the value $p_{e}=\frac{5}{6}$ which is the solution when there is no natural selection. When $\theta=0$, then of course $p_{e}=0$ since $\Delta p=\frac{-s p q}{1-s(p-q)}$. The equilibrium values lie in the range $0 \leqq p_{e} \leqq \frac{5}{6}$. They are stable equilibria since at $p=p_{e}, d(\Delta p) / d p<0$.

Unfortunately, when natural selection acts only on the males, the corresponding set of equilibrium values cannot be obtained, explicitly, since the recurrence equations contain both $p$ and $u$. 
(iv) Natural selection with frequency-dependent sexual selection

When the sexual selection is partly frequency-dependent, explicit solutions to the recurrence equations can be found for some of the more interesting models. If the mating preferences are partly dependent on the frequencies of the genotypes, then as before we may put

so that

$$
\begin{aligned}
& \alpha=a+k u \\
& \beta=b+k v \\
& \gamma=c+k w
\end{aligned}
$$

$$
\begin{aligned}
& \theta=a+b+c+k \\
& \phi=a+\frac{1}{2} b+k p
\end{aligned}
$$

and for an allele with additive effects

$$
\Delta p \simeq \frac{1}{2}\left(a+\frac{1}{2} b\right)-\frac{1}{2}(a+b+c) p-s p q .
$$

Thus the frequency-dependent component of the sexual selection has no effect for there is no term containing $k$. If $b=\frac{1}{2} a$ and $c=0$, then

$$
\Delta p \simeq \frac{5}{8} a-\frac{3}{4} a p-s p q
$$

with an equilibrium at the value

$$
p_{e}=\frac{1}{2}\left\{1+\frac{8}{4}\left(\frac{a}{s}\right)-\sqrt{1-\left(\frac{a}{s}\right)+\frac{9}{16}\left(\frac{a}{s}\right)^{2}}\right\} .
$$

This is the same solution as when mating preferences are constant except that $\theta=\frac{3}{2} a$, which is now the constant part of the mating preference.

In the more general model of frequency-dependent mating preferences, we have

$$
\begin{aligned}
& \alpha=a+k u \\
& \beta=b+l v \\
& \gamma=c+m w .
\end{aligned}
$$

If the allele is additive in respect of the mating preferences $c=m=0$, $b=\frac{1}{2} a$ and $l=\frac{1}{2} k$, so that

$$
\begin{aligned}
\theta & =\frac{3}{2} a+k p \\
\phi & =\frac{5}{4} a+\frac{1}{2} k(p+u) \\
& =\frac{5}{4} a+\frac{1}{2} k p(1+p)
\end{aligned}
$$

if we put $u=p^{2}$ as an approximation. The recurrence equation is now approximately

$$
\Delta p \simeq \frac{5}{8} a-\frac{3}{4} a p-p q\left(s-\frac{1}{4} k\right)
$$

which has a similar solution except that $s$ must be replaced by $s-1 k$. These results show that when an allele has additive effects its equilibrium frequency is found from the expression

$$
p_{e}=\frac{1}{2}\left\{1+\frac{3}{4}\left(\frac{a}{x}\right)-\sqrt{1-\left(\frac{a}{x}\right)+\frac{9}{16}\left(\frac{a}{x}\right)^{2}}\right\},
$$


where $x$ may be either the simple natural selective coefficient $s$, or the expression $s-\frac{1}{4} k$, depending on how the mating preference varies with frequency. Given any gene frequency at equilibrium, the value of $a / x$ required to produce it can be calculated. If $x$ is negative, then the sexual selection will always produce fixation of the allelle.

\section{SeXual selection In sticklebacks}

Semler (1971) studied the mating preferences of female Threespine sticklebacks (Gasterosteus aculeatus) for males who develop the characteristic red throat colour at breeding time. Some populations are polymorphic for this character with both red and non-red breeding males. These populations have been found in lakes in the North-western United States where the Rainbow trout (Salmo gairdneri) and Cutthroat trout (Salmo clarkii) prey heavily on the sticklebacks. Moodie (1972) has shown that trout attack red sticklebacks many times more often than they attack non-red ones. The red males must therefore be at a great selective disadvantage as a result of the predation. But they can get more females to spawn in their nests and must have an advantage in sexual selection.

Semler tested single females with a red and a non-red male in a series of trials. He found there was no significant difference between artificially coloured and naturally coloured males in the proportions in which they were chosen by the females. His combined data show that 38 females spawned in nests of red males and only 15 spawned in nests of non-red males. Thus if a proportion $\alpha$ of these females have exercised a mating preference for red males, then the expected proportion of females mating with red males is $\alpha+\frac{1}{2}(1-\alpha)=\frac{1}{2}(1+\alpha)$ and the expected proportion mating with the others is $\frac{1}{2}(1-\alpha)$. Thus we obtain the estimate

$$
\alpha=23 / 53=0.434 \text {. }
$$

This is the maximum likelihood estimate with a standard error of $0 \cdot 124$.

Unfortunately, the genetics of the polymorphism is not clearly understood: crosses between black and red parents apparently produce intermediate and rather variable progeny. The polymorphism may be controlled by a major gene with a number of modifiers. In Wapato Lake in Eastern Washington, the frequency of the red males is around 14 per cent. to 18 per cent. at the start of the breeding season and it drops to around 5 per cent. after two months. If 5 per cent. is the equilibrium frequency after selection, then the natural selective coefficient required to maintain it can be calculated by equating it to a theoretical equilibrium frequency given by an appropriate model. Suppose the polymorphism is caused by a pair of alleles with additive effects. At equilibrium with constant mating preferences the gene frequency is

$$
p_{e}=\frac{1}{2}\left\{1+\frac{1}{2}\left(\frac{\theta}{s}\right)-\sqrt{1-\frac{2}{3}\left(\frac{\theta}{s}\right)+\frac{1}{4}\left(\frac{\theta}{s}\right)^{2}}\right\} .
$$

After both mating and natural selection has taken place the genotypic frequencies are as follows.

$$
\begin{aligned}
T u_{e}^{\prime} & =(1-s)\left\{\frac{5}{6} \theta p_{e}+(1-\theta) p_{e}^{2}\right\} \\
T v_{e}^{\prime} & =\frac{5}{6} \theta q_{e}+\frac{1}{6} \theta p_{e}+2(1-\theta) p_{e} q_{e} \\
T w_{e}^{\prime} & =(1+s)\left\{\frac{1}{6} \theta q_{e}+(1-\theta) q_{e}^{2}\right\} .
\end{aligned}
$$


If the genes are acting additively, the heterozygotes, at a frequency $v_{e}^{\prime}$ after natural selection, will be phenotypes with less red colour than the homozygous red males at a frequency $u_{e}^{\prime}$. The frequency of males with some red colour will therefore be $u_{e}^{\prime}+v_{e}^{\prime}$. In his experiments, Semler used any male with red colour. Hence we may put $\theta=0.434$ to find the value of $s$ that gives a value of $u_{e}^{\prime}+v_{e}^{\prime}=0.05$. When $s=1$, its greatest possible value, we get $u_{e}^{\prime}+v_{e}^{\prime}=0.347$, which is much too high. If we take the lower 95 per cent. confidence limit of $\theta(\theta=0.186)$ we get when $s=1 u_{e}^{\prime}+v_{e}^{\prime}=0.152$, which is again too high. This calculation also assumes that natural selection affects both males and females. If it affects only the males, when very high values of $s$ giving negative fitnesses would be necessary to produce the observed equilibrium frequency. Even when the lower confidence limit is taken for $\theta$, the model is not therefore a satisfactory fit to the data; though in nature $\theta$ may well be lower than in Semler's experiments in which the females did not have to face any competition for mates themselves.

If either a recessive or a dominant gene determines the red throat, however, the model then does give a reasonable value for $s$. When natural selection affects only the males, the red males have an equilibrium frequency of $\alpha / s$ before the selection and $\frac{\alpha(1-s)}{s(1-\alpha)}$ after it. Thus we may put

$$
\frac{\alpha(1-s)}{s(1-\alpha)}=0.053 \text { (the frequency observed by Semler) }
$$

which gives $s=0.935$ with upper and lower confidence limits of 0.812 and 0.976 . These are high but reasonable values, for Semler observed that the frequency of the red males was reduced from 0.13 to 0.05 in the first 2 months of the breeding season. If all the natural selection had then taken place we should have $s=0.65$. But in fact the breeding period lasts for about 5 months so the over-all selective coefficient is likely to be very high indeed.

Comment added in proof.-Dr. D. W. Hagen has pointed out to me that black is a distinct phenotype different from non-red. There is complete dominance at the non-red locus.

\section{REFERENCES}

COOCH, F. G., AND BEARDMORE, J. A. 1959. Assortative mating and reciprocal difference in the Blue-Snow Goose complex. Nature, 183, 1833-1834.

DARwin, c. 1871. The Descent of Man and Selection in Relation to Sex. London.

FIsHer, R. A. 1930. The Genetical Theory of Natural Selection. Clarendon Press, Oxford.

KALMUS, H., AND MAYNARD SMITH, s. 1966. Some evolutionary consequences of pegmatypic mating systems (imprinting). The American Naturalist, 100, 619-635.

KARLIN, s. 1969. Equilibrium Behavior of Population Genetic Models with Non-random Mating. Gordon and Breach, Science Publishers, New York.

o'DONALD, P. 1962. The theory of sexual selection. Heredity, 17, 541-552.

o'Donald, P. 1963. Sexual selection for dominant and recessive genes. Heredity, 18, 451-457. o'Donald, P. 1967. A general model of sexual and natural selection. Heredity, 22, 499-518. o'DONALD, P. 1972a. Natural selection of reproductive rates and breeding times and its effect on sexual selection. The American Naturalist, 106, 368-379.

o'Donald, P. 1972b. Sexual selection by variations in fitness at breeding time. Nature, 237, 349-351.

o'Donald, P. 1972c. Sexual selection for colour phases in the Arctic Skua. Nature, 238, 403404.

MOODIE, G. E. E. 1972. Predation, natural selection and adaptation in an unusual Threespine stickleback. Heredity, 28, 155-167.

SEMLER, D. E. 1971. Some aspects of adaptation in a polymorphism for breeding colours in the Threespine stickleback (Gasterostetus aculeatus). F. Zool., Lond., 165, 291-302. 